\title{
The Design of Cleaning Robot Based on ARM Microprocessor
}

\author{
Dan Li \\ College of Electrical and Information Engineering, Jilin Agricultural Science and Technology \\ University, Jilin City, China \\ 653301981@qq.com
}

Keywords: Cleaning robot; Microprocessor; Sensor system; Wireless communication

\begin{abstract}
This paper describes the design of household intelligent cleaning robot, which is an innovative work of collegiate contest. This paper describes the general structure of system, hardware circuit design and software program design. The robot can achieve autonomous movement, garbage cleaning, obstacle avoidance and other functions. It can work in the home, library, exhibition halls and other indoor environment. This design, which is applied to the training of electronic course group, not only cultivates the students' professional interest but also improves the students' engineering practice ability and innovation ability. This design has been successfully used in the electronic course group training of application-oriented universities.
\end{abstract}

\section{Introduction}

With the development of computer technology and artificial intelligence technology, smart home now has become a hot topic in the electronics industry. The use of robots gets people's attention. Service robot has infiltrated every aspect of our lives and works. In order to complete the people liberated from household cleaning, further study of cleaning robot becomes more and more important. [1]

This paper chooses the design of household intelligent cleaning robot as electronic science college competition work. Using the college students competition reforms curriculum system, improves the experimental equipment, cultivates the students' professional interest, engineering practice ability and innovation ability, changes the way students' working, thinking, working efficiency and collaboration capabilities. [2]It is supported by Higher Education Scientific Research Project of Jilin Provincial Institute for Higher Education(JGJX2015C90).

\section{The Overall Design of the Cleaning Robot}

Intelligent cleaning robot moves in indoor environment to realize the function such as cleaning, obstacle avoidance and path planning. With regulation and control of all system components, it traverses every corner as possible to achieve sweeping intelligence. Robot time recording function can implement scheduled cleaning tasks. After cleaning tasks, it can automatically return to the starting point. Robot combines its own perception and control functions to realize the path planning and obstacle avoidance, and other functions. As robot cleaning function is perfect, in the process of movement, it collects the garbage on the ground together, and picks them up to the litter box. Cleaning robot top view is shown in Fig. 1, and cleaning robot bottom view is shown in Fig. 2.

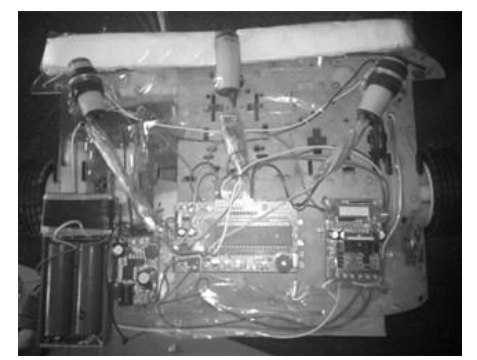

Figure 1. Cleaning robot top view

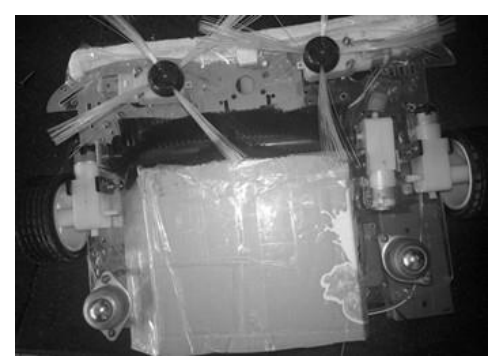

Figure 2. Cleaning robot bottom view 


\section{The System Composition of the Cleaning Robot}

Household intelligent cleaning robot is mainly composed of four parts: the motion system, the sensor system, the control system and the cleaning system. Motion system is the important part of a cleaning robot, which determines the robot movement space and cleaning effect. Robot-driven design is two-wheel-drive, which can be divided into front and rear-wheel drive. The benefit of front-wheel drive is that steering performance is improved. While rear wheel is turning wheel, driving direction is easy to control and not prone to excessive steering, so that steering safety is improved. [3]

In order to avoid obstacles in cleaning route, the cleaning robot must use the sensing system to real-time monitor the position, status, movement environment, to ensure that the cleaning robot is in a normal operating state. The sensors systems of the robot are divided into internal sensors and external sensors. Internal sensors include rotary encoders and gyroscopes. Rotary encoders can be used to calculate the robot's movement speed, acceleration, and its location. They can be used to judge the posture of cleaning robot (the angle between the direction of movement and the wheels). They make it easier to control movement. [4]External sensors include visual sensors, ultrasonic sensors, infrared sensors, and proximity sensor. Visual sensors use CCD camera for robot visual navigation, positioning, target recognition and map construction. Ultrasonic sensors measure distance among obstacles in the working environment and make the map construction. Infrared sensors mostly use infrared proximity switches to detect obstacles in the working environment and avoiding collision. Proximity sensors are used for obstacle avoidance.

Control system is the core part of the robot, which receives information passed by sensors system and other parts, and analyzes the information, so as to control the robot cleaning, moving, stopping, charging, and other functions. [5]The control system makes the robots more intelligent. User can also press on the buttons of the robot to control the robot's behavior, such as cleaning time and charging time. Control system uses microprocessor S3C44B0X to deal with the data for controlling the robot's movement speed, trajectory, work status, and so on.

The robot cleaning work is done by the cleaning system, combining suction part and sweep part. The cleaning system uses the cleaning brush to swept garbage to the chassis of the robot, then vacuuming junk inhaled garbage into the collector. Cleaning robot can also complete the work smoothly, although there is large garbage. When encountering failures, the robot will use warning lights to send warning. If the garbage collector is full of junk inside, the robot will automatically return to the starting location, waiting for people to pour out our trash.

According to the robot functional requirements analysis and system analysis, system overall design is shown in Fig. 3.

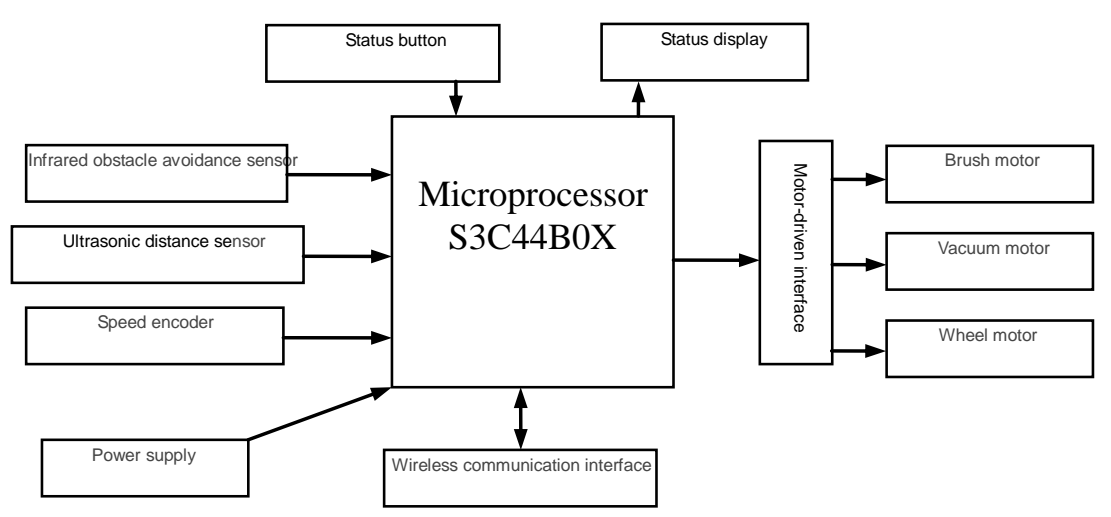

Figure 3. The overall design of robot system

\section{The Hardware Design of the Cleaning Robot}

The Design of the Control Core Module. Cleaning robot control system uses microprocessor S3C44B0X as the core, which can be responsible for receiving and transmitting instructions, 
controlling motor steering, cleaning, obstacle avoidance, and other functions. As shown in Fig. 3, the processor is connected to several modules, such as wireless communication interface, infrared obstacle avoidance sensor, ultrasonic distance sensor, speed encoder, motor-driven interface, status button, status display, etc. These modules are controlled by the microprocessor to co-ordinate work, and ensure the realization of cleaning robot features. [6]

Cleaning robot control system uses Samsung's high performance-to-price ratio and high performance 16/32 bit RISC microprocessor S3C44B0X chips. S3C44B0X uses ARM7TDMI core, 0.25 um CMOS standard unit memory. [7]S3C44B0X also provides the following parts: $8 \mathrm{~KB}$ Cache, optional internal SRAM, 2 channels UART, 4 channel DMA, 8 channels of the 12 bit ADC channels, the I2C/I2S bus interface, synchronous SIO interface and pairs of clock PLL, and other resources.

Because it does not have internal FLASH, S3C44B0X puts FLASH Chip MBM29LV160T into the system. According to the intrinsic characteristics of the S3C44B0X, it takes Pin nGCS0 as a FLASH optional port to make FLASH addresses start from 0X00000000.The system uses HY57V641620 chip as external DRAM of S3C44B0X. According to the intrinsic characteristics of the S3C44B0X, it takes Pin nGCS6 as a DRAM optional port to make DRAM addresses start from 0X0C000000.

The Structure Design of Robot Body. In order to meet the performance requirements of robot, the robot's mechanical structure should have stability, flexibility, adequate strength. At the same time, it should have smaller size, larger capacity, and lighter weight. Cleaning robot's mechanical structure is mainly composed of outer shell, the car chassis, driving wheel, sensor, cleaning module, etc.[8]

The external body and chassis adopt aluminum alloy frame to increase the firmness and to reduce weight. Sensors are installed in the appropriate place in the car and connected by wires, so that the robot reduces the vehicle center of gravity to improve the stability of car.

Garbage cleaning module is composed of a garbage collecting device and a cleaning module. The garbage collecting device is installed on the middle part of the chassis. Cleaning module is divided into sweeping module and vacuum device module. Sweeping and vacuum were put in the chassis front ends and the garbage collector. [9]

The Design of Wireless Communication Interface. Wireless communication interface module function is to complete the communication between the cleaning robot and charging station. Wireless communication chip nRF905 interface circuit schematic diagram shown in Fig. 4 [10]

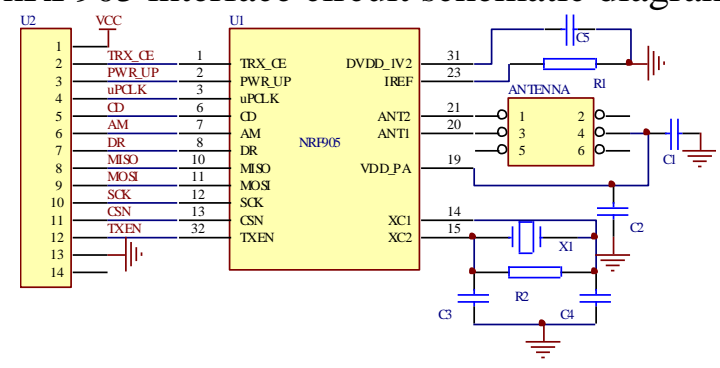

Figure 4. NRF905 interface circuit schematic diagram

\section{Summary}

By studying the design and debugging the cleaning robot, it is proved that the service robot brings convenience to people. The cleaning robot not only saves manpower and material resources, but also creates a good living environment. In the popularization and application process of cleaning robot with a low price, there will be huge market prospects. Service robots in the future will be greater demand. The application of intelligent cleaning robot will replace heavy cleaning tools on the market. It has opened up a new era of intelligent cleaning. 


\section{Acknowledgements}

This paper is supported by Higher Education Scientific Research Project of Jilin Provincial Institute for Higher Education (JGJX2015C90) and supported by Jilin Province education science 12th Five-Year foundation (GH14335).

This paper is supported by Youth Fund Project of Jilin Agricultural Science and Technology University (NO.2015224).

This paper is supported by Key Subject Cultivation Project of Jilin Agricultural Science and Technology University (NO.2015X093).

\section{References}

[1] Seongsoo Lee: Vision-Based Kidnap Recovery with SLAM for Home Cleaning Robots, Journal of Intelligent \& Robotic Systems, Vol. 67(2012) No. 1, p.7.

[2] J.G.Jiang:Implementation of glass-curtain-wall cleaning robot driven by double flexible rope, The Industrial Robot,Vol.41(2014)No.5,p.438.

[3] Tae-Kyeong Lee:Sector-based maximal online coverage of unknown environments for cleaning robots with limited sensing, Robotics and Autonomous Systems, Vol.59(2011)No.10, p.698.

[4] Ding Feng:Lecture Notes in Computer Science (Springer Berlin Heidelberg, Germany 2008 ), p.20.

[5] Man Hyung Lee:On the synthesis of an underwater ship hull cleaning robot system, International Journal of Precision Engineering and Manufacturing, Vol.13(2012)No.11, p.1965.

[6] H.Y.Liu:Design and Implementation of an Intelligent Cleaning Robot Based on Fuzzy Control, Advanced Materials Research, Vol.3401(2014)No.1003, p.221.

[7] J.L.Zhang:Comprehensive obstacle avoidance system based on the fuzzy control for cleaning robot, Machine Tool \& Hydraulics, Vol.18(2014), p.92.

[8] Houssam Albitar:In-water surface cleaning robot: concept, locomotion and stability, Int. J. of Mechatronics and Automation, Vol.4(2014)No.2, p.104.

[9] Haiyan Sun: Lecture Notes in Electrical Engineering (Springer Berlin Heidelberg, Germany 2012), p.10.

[10]Brett Stern:Helen Greiner(Apress, America 2012), p.1. 